\title{
Chrysin-induced ERK1/2 Phosphorylation Enhances the Sensitivity of Human Hepatocellular Carcinoma Cells to Sorafenib
}

\author{
CHING-TING WEI ${ }^{1,2}$, LEI-CHIN CHEN $^{3}$, YI-PING HSIANG ${ }^{4}$, YUNG-JUN HUNG ${ }^{4}$, \\ PEI-HSUAN CHIEN ${ }^{5}$, HSIAO-LIN PAN ${ }^{5}$ and YUN-JU CHEN ${ }^{1,5,6}$ \\ ${ }^{1}$ School of Medicine for International Students, I-Shou University, Kaohsiung, Taiwan, R.O.C.; \\ ${ }^{2}$ Division of General Surgery, Department of Surgery, E-Da Hospital, Kaohsiung, Taiwan, R.O.C.; \\ ${ }^{3}$ Department of Nutrition, I-Shou University, Kaohsiung, Taiwan, R.O.C.; \\ ${ }^{4}$ Department of Pharmacy, E-Da Hospital, Kaohsiung, Taiwan, R.O.C.; \\ ${ }^{5}$ Department of Medical Research, E-Da Hospital, Kaohsiung, Taiwan, R.O.C.; \\ ${ }^{6}$ Department of Pharmacy, E-Da Cancer Hospital, Kaohsiung, Taiwan, R.O.C.
}

\begin{abstract}
Background/Aim: Sorafenib is now standard treatment for advanced hepatocellular carcinoma (HCC). However, therapeutic efficacy is not as good as was predicted. Many efforts are being made to improve HCC sensitivity to sorafenib. Our previous study demonstrated that co-treatment with chrysin enhanced sorafenib sensitivity through inhibition of ATP-binding cassette superfamily $G$ member 2 (ABCG2). Whether there is another mechanism other than inhibition of ABCG2 underlying chrysin-mediated synergistic effect is still not completely elucidated. Materials and Methods: Phosphorylation of extracellular signal-regulated protein kinases 1 and 2 (ERK1/2) was examined by western blot. Cell viability was examined by crystal violet staining. The importance of ERK1/2 phosphorylation was assessed by overexpression and blockage of mitogen-activated protein kinase kinase 1 (MEK1). Results: Chrysin induced sustained ERK1/2 phosphorylation of HCC cells in both time- and dosedependent manners. Overexpression of MEKI enhanced, whereas blockage of MEK1 led to loss of chrysin-synergized sorafenib effect, through modulating ERK1/2 phosphorylation level. Conclusion: These results identify another novel mechanism underlying chrysin-mediated synergistic effect on sorafenib activity in HCC cells.
\end{abstract}

Correspondence to: Yun-Ju Chen, School of Medicine for International Students, I-Shou University, No. 8, Yida Rd., Jiaosu Village, Yanchao District, Kaohsiung City 82445, Taiwan, R.O.C. E-mail: yjchen0326@isu.edu.tw

Key Words: HCC, chrysin, sorafenib.
Sorafenib (Nexavar ${ }^{\circledR}$, BAY43-9006), is an orally active multikinase inhibitor targeting not only tumor cell proliferation, through inhibition of rapidly accelerated fibrosarcoma (RAF)-dependent signaling pathway, but also tumor angiogenesis via blockage of vascular endothelial growth factor receptors, platelet-derived growth factor receptor $\beta$, FMS-like tyrosine kinase 3 , c-KIT protein as well as RET receptor tyrosine kinase $(1,2)$. Since advanced hepatocellular carcinoma (HCC) is characterized by strong angiogenesis and sorafenib is well tolerated in HCC, sorafenib is now the only drug approved by the US Food and Drug Administration for the standard treatment of advanced HCC. However, the therapeutic response is not as predicted $(3,4)$. Many efforts are being made to elucidate the mechanisms underlying resistance to sorafenib therapy and to develop strategies for maximizing its treatment efficacy. Our previous study demonstrated that ATP-binding cassette super-family G member 2 (ABCG2), well-known as being involved in drug efflux and multidrug resistance to cancer chemotherapy $(5,6)$, mediated the efflux of sorafenib by $\mathrm{HCC}$ cells and in turn attenuated its antitumor growth effect. Co-treatment with chrysin, an inhibitor of ABCG2, greatly augmented the antitumor activity of sorafenib in HCC cells. Therefore, ABCG2 is a potential predictor for sorafenib sensitivity in HCC and blockage of its activity is a strategy to increase sorafenib efficacy in HCC cells (7).

Chrysin (also known as 5,7-dihydroxyflavone) is a naturallyoccurring flavonoid with biological activity and is commonly found in food. Among them, propolis particularly has the most abundance $(8,9)$. In addition to acting as an inhibitor of ABCG2, chrysin per se is reported to have antitumor activities in several cancer types $(10,11)$. The underlying mechanism includes the involvement of cell-cycle arrest and apoptosis (11, 
12). Our recent study indicated that chrysin attenuated cell viability of human colorectal cancer cells through induction of autophagy (13). In the present study, we further investigated whether there are mechanisms other than inhibition of drug efflux underlying chrysin-mediated synergistic effects on sorafenib activity in HCC cells.

\section{Materials and Methods}

Cell lines and reagents. Hep3B and HepG2 HCC cells from the American Type Culture Collection (Manassas, VA, USA) were maintained in Dulbecco's modified Eagle's medium/F12 medium supplemented with $10 \%$ fetal bovine serum (FBS) (Logan, UT, USA). Sorafenib was kindly provided by Dr. Chao-Ming Hung (EDa Hospital, Kaohsiung, Taiwan, ROC) and was dissolved in dimethyl sulfoxide (DMSO) as stock concentration at $100 \mu \mathrm{M}$. Chrysin, DMSO, crystal violet, mitogen-activated protein kinase kinase 1 (MEK1) inhibitor U0126, 3-(4,5-dimethylthiazol-2-yl)-2,5diphenyltetrazolium bromide (MTT), control small interfering RNA (siRNA), c-RAF small interfering RNA (siRNA), MEK1 siRNA and antibodies against tubulin, hemagglutinin (HA) tag as well as actin were from Sigma-Aldrich (St. Louis, MO, USA). Antibodies against phospho-extracellular signal-regulated protein kinases 1 and 2 (ERK1/2)-Thr202/Tyr204, c-RAF and MEK1 were purchased from Cell Signaling (Danvers, MA, USA). ERK1/2 antibody was from Millipore (Burlington, MA, USA). TransIT-2020 transfection reagent was purchased from Mirus Bio LLC (Madison, WI, USA).

Transfection assay. For qualitative DNA transfection, $1 \mu \mathrm{g}$ DNA was transfected into HCC cells with $70-80 \%$ confluence in a $6-\mathrm{cm}$ dish using $1 \mu \mathrm{l}$ TransIT-2020 transfection reagent. One day later, cells at a density of $1 \times 10^{5}$ cells/well were re-seeded on 6-well plates then experiments were carried out as described below. For qualitative siRNA transfection, a final concentration of $100 \mathrm{nM}$ siRNA was transfected into HCC cells with $70-80 \%$ confluence in a 6-cm dish by using $1 \mu \mathrm{l}$ TransIT-2020 transfection reagent. Two days later, cells at a density of $1 \times 10^{5}$ cells/well were re-seeded on 6-well plates and experiments were carried out as described below.

In vitro cell viability assay. In vitro quantitative cell viability assay was evaluated using the MTT colorimetric assay or crystal violet staining. For MTT assay, HCC cells at a density of $5 \times 10^{3}$ cells/well were seeded on 96-well plates overnight. Then cells were subjected to pre-treatment with vehicle control (DMSO) or $10 \mu \mathrm{M}$ U0126 for 1 hour followed by individual treatments with vehicle control (DMSO), $5 \mu \mathrm{M}$ sorafenib or $5 \mu \mathrm{M}$ sorafenib plus $25 \mu \mathrm{M}$ chrysin for 2 days. Subsequently, $1 \mu \mathrm{g} / \mathrm{ml}$ MTT was added to each well. The medium was removed after 4-hour incubation and formazan was solubilized in $100 \mu \mathrm{l}$ DMSO per well, followed by the measurement of absorbance at $570 \mathrm{~nm}$ for relative cell viability.

HCC cells were seeded at a density of $5 \times 10^{3}$ cells/well, followed by treatment with vehicle control DMSO or $25 \mu \mathrm{M}$ chrysin. Relative cell growth rate was examined at day 1,2 , and 3 by MTT assay.

For crystal violet staining, HCC cells at a density of $1 \times 10^{5}$ cells/well were seeded on 6-well plates overnight. Subsequently, cells were subjected to individual treatments with vehicle control DMSO, $5 \mu \mathrm{M}$ sorafenib, $25 \mu \mathrm{M}$ chrysin or $5 \mu \mathrm{M}$ sorafenib plus $25 \mu \mathrm{M}$ chrysin for 2 days. Cells were then washed with $1 \times$ phosphate-buffered saline twice and fixed, followed by staining with $1 \%$ crystal violet dissolved in 30\% ethanol for 15-30 minutes at room temperature. Next, cells were washed with tap water twice in order to eliminate background interference. The crystal violet-stained plates were air dried and subjected to photography and quantification.

For analysis of the effect of FBS concentration on chrysinsynergized sorafenib activity, HCC cells at a density of $1 \times 10^{5}$ cells/well were seeded and maintained in medium supplemented with different FBS concentrations overnight. Similar procedures for drug treatments and crystal violet staining described above were then performed.

Western blot. HCC cells were treated with $25 \mu \mathrm{M}$ chrysin for different time periods or treated with different concentrations of chrysin for 24 hours. The whole cell lysates were then harvested, and subjected to qualitative western blot procedure, including electrophoresis, gel transfer and blocking. Protein expressions were examined using antibodies against pp-ERK1/2 Thr202/Tyr204 $(1: 1,000)$, ERK1/2 $(1: 5,000)$ and actin $(1: 10,000)$. Whole cell lysates from HA-MEK1 DNA transfection for 2 days, si-control/sic-RAF/si-MEK1 transfection for 4 days or treatment with U0126 for 1 hour were individually harvested, and subjected to western blot procedure described above. Protein expressions were examined using antibodies against pp-ERK1/2 Thr202/Tyr204 (1:1000), ERK1/2 (1:5000), actin (1:10000), tubulin (1:10000), HA tag (1:5000), c-RAF (1:1000) and MEK1 (1:1000).

Statistical analysis. The statistical analysis was performed using Student $t$-test. $p$-Values of less than 0.05 were considered statistically significant.

\section{Results}

Chrysin induced time- and dose-dependent sustained phosphorylation of ERK1/2 in HCC cells. We first examined whether chrysin increases ERK1/2 phosphorylation of HCC cells to enhance sorafenib anti-tumor effects. HCC cells were treated for different time periods and dosages of sorafenib and the results showed that chrysin induced sustained phosphorylation of ERK1/2 in Hep3B HCC cells and such ERK1/2 phosphorylation lasted for 24 hours (Figure 1A). Similar results were observed in HepG2 HCC cells (Figure 1B). Moreover, when the concentration of chrysin was increased, the level of ERK1/2 phosphorylation was gradually enhanced in both Hep3B and HepG2 cells (Figure $1 \mathrm{C}$ and $\mathrm{D})$. These results suggest that chrysin induces sustained ERK1/2 phosphorylation of HCC cells in time- and dose-dependent manners.

ERK1/2 phosphorylation was required for chrysin-mediated synergistic effect on sorafenib activity in HCC cells. We further investigated the importance of ERK $1 / 2$ phosphorylation in chrysin-synergized inhibition of cell viability by sorafenib in HCC cells. To this end, induction of ERK1/2 phosphorylation by overexpression of its upstream regulator, MEK1 as well as inhibition of ERK1/2 phosphorylation by MEK1 inhibitor U0126 and MEK1 siRNA were applied. As shown in Figure $2 \mathrm{~A}$, in the vector control group, sorafenib hardly attenuated cell viability of Hep3B cells, but clearly inhibited cell viability in 
A

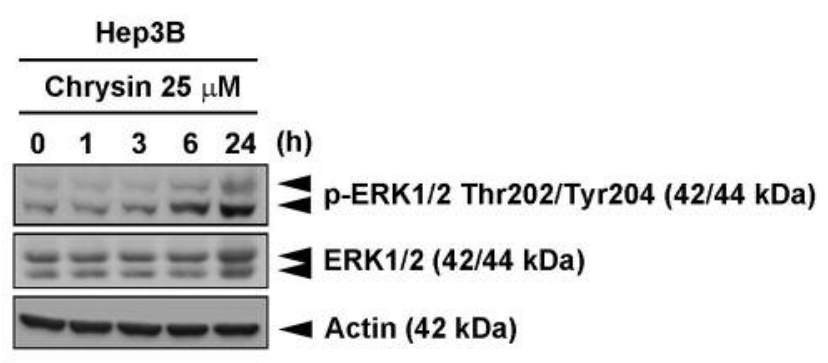

B

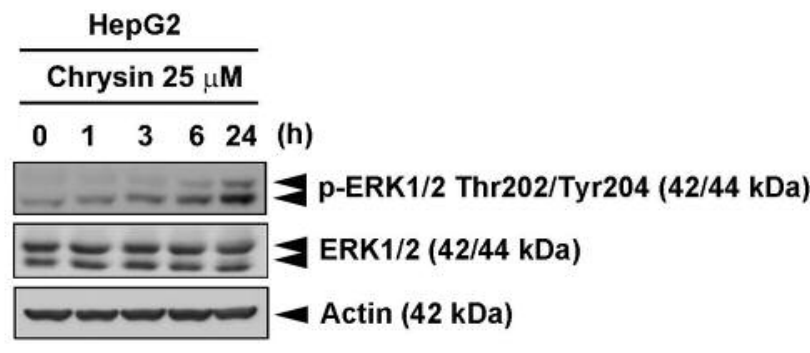

C

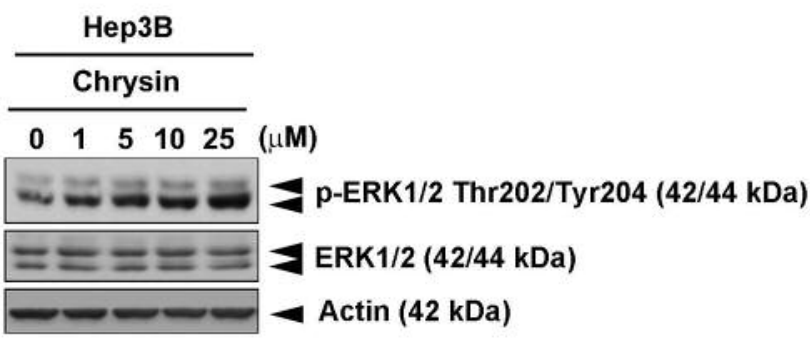

D

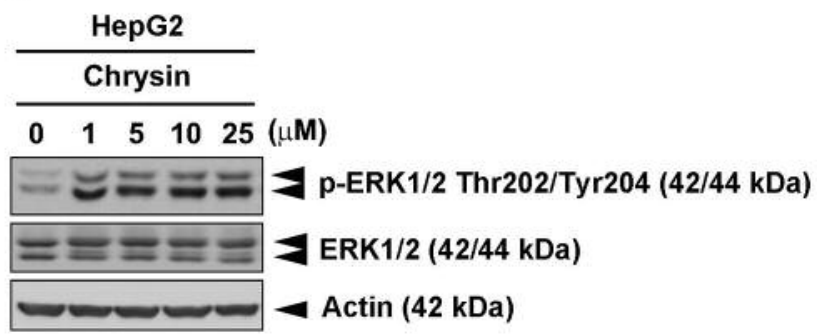

Figure 1. Chrysin induced time- and dose-dependent phosphorylation of extracellular signal-regulated protein kinases 1 and 2 (ERK1/2) in hepatocellular carcinoma (HCC) cells. Hep3B and HepG2 HCC cells were treated with $25 \mu M$ chrysin for different time periods (A and B, respectively) or with different concentrations of chrysin for 24 hours ( $C$ and $D$, respectively) and then the whole-cell lysates were harvested and subjected to western blot. Protein expressions were examined using antibodies to phospho-ERK1/2 (p-ERK1/2)-Thr202/Tyr204, ERK1/2 and actin.

cells co-treated with chrysin. However, when HA-MEK1 was overexpressed in cells, ERK1/2 phosphorylation was increased (Figure 2A, lower panel) and chrysin-synergized inhibition of cell viability by sorafenib was further slightly enhanced (Figure 2A, upper panel). On the other hand, when ERK1/2 phosphorylation in Hep3B cells was inhibited by U0126 (Figure 2B, right panel), chrysin-enhanced inhibition of cell viability by sorafenib was somewhat abrogated as compared to that in the control group (Figure 2B, left panel). Similar results were observed in HepG2 cells (Figure 2C). In concordance with this result, when MEK1 expression in HepG2 cells was silenced by its siRNA (Figure 2D, right panel), the cell viability inhibition by sorafenib and chrysin combination was slightly reversed as compared to that in si-control group (Figure 2D, left and middle panels). Interestingly, such inhibition of cell viability by sorafenib and chrysin combination observed in the si-control group was not affected when the expression of MEK1 upstream regulator, c-RAF was removed (Figure 2D), implying that a c-RAF-independent mechanism may be involved in this regulation. Collectively, these results suggest that ERK1/2 phosphorylation is required for chrysin-mediated synergistic effect on sorafenib activity in HCC cells.

A declining growth pattern through chrysin-mediated sustained ERK1/2 phosphorylation conferred sensitivity of
HCC cells to sorafenib treatment. Next, we explored the reason why sustained ERK1/2 phosphorylation enhances the synergistic effect on sorafenib activity by chrysin in HCC cells. It is known that the RAF-MEK-ERK1/2 pathway transduces the mitogenic signal step by step to induce cell proliferation. It is worth mentioning that these signaling molecules, including ERK1/2, usually display transient phosphorylation in order to transmit the signal to next one. However, it is reported that if ERK1/2 phosphorylation is maintained, cells will turn to differentiation instead of proliferation (17-20). Thus, we examined the effect of chrysin on the growth rate of HCC cells. The results showed that the growth rates of Hep3B and HepG2 cells with chrysin treatment were slightly less than that of cells without chrysin treatment (Figure 3A). To mimic the scenario of a lessproliferative state, different percentages of serum in cell culture medium were used. As shown in Figure 3B, the cell growth was largely slow when the percentage of serum in culture medium of Hep3B and HepG 2 cells was reduced from $10 \%$ to $1 \%$. We also examined the ERK $1 / 2$ phosphorylation level under these conditions and found that Hep3B and HepG 2 cells maintained in culture medium with $1 \%$ serum presented a higher level of ERK1/2 phosphorylation than that in cells with $10 \%$ serum (Figure 3C). We next investigated the sensitivity of cells to sorafenib treatment in culture 
A
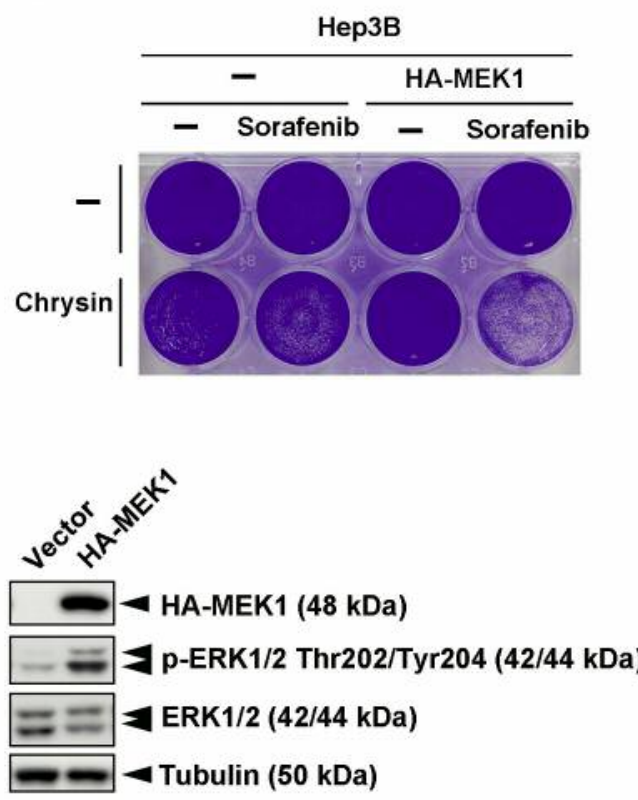

B

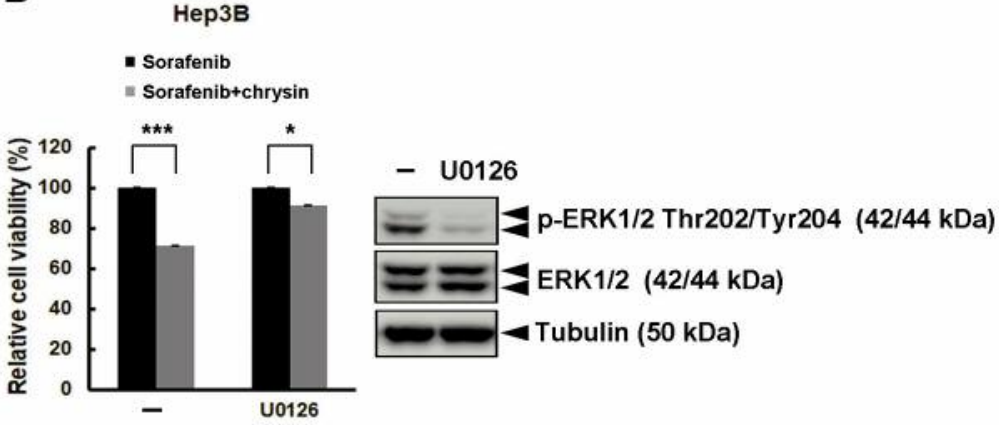

C

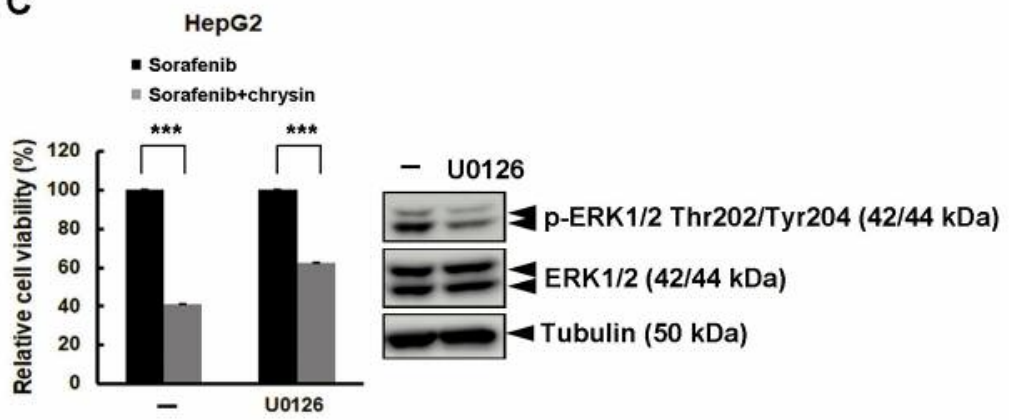

D

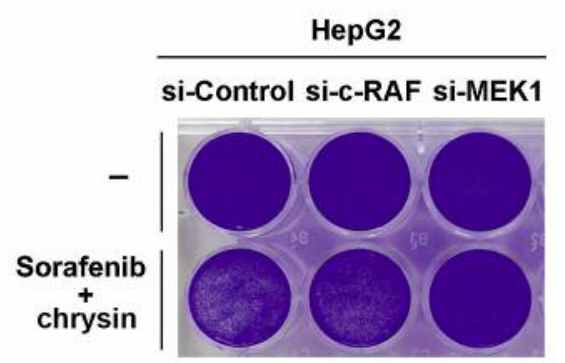

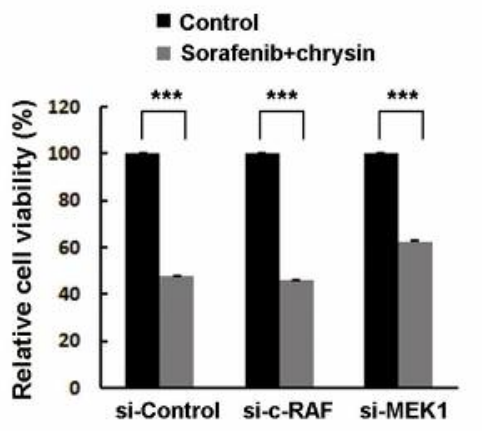

Figure 2. Phosphorylation of extracellular signal-regulated protein kinases 1 and 2 (ERK1/2) is important for chrysin-mediated synergistic inhibition of cell viability by sorafenib in hepatocellular carcinoma (HCC) cells. Hep3B cells were transfected with or without hemagglutinin-tagged mitogenactivated protein kinase kinase 1 (HA-MEK1) expression vector for $24 \mathrm{~h}(\mathrm{~A})$. Cells in both groups were re-seeded at a density of $1 \times 10^{5}$ cells $/$ well, followed by treatment with $25 \mu \mathrm{M}$ chrysin and $5 \mu \mathrm{M}$ sorafenib for 2 days. The cell viability was examined by crystal violet staining (upper panel). HA-MEK1 expression was examined by western blot (lower panel). Hep3B (B) and HepG2 (C) cells were pre-treated with $10 \mu M$ MEK1 inhibitor U0126 for $1 \mathrm{~h}$, followed by treatment of $25 \mu \mathrm{M}$ chrysin and $5 \mu \mathrm{M}$ sorafenib for 2 days. Cell viability was examined by crystal violet staining and statistically quantified (left panel) and ERK1/2 phosphorylation level was examined by western blot (right panel). D: HepG2 cells were separately transfected with small-interfering (si)-control, si-c-rapidly accelerated fibrosarcoma (RAF) and si-MEK1. Two days later, cells in each group were re-seeded at a density of $1 \times 10^{5} \mathrm{cells} /$ well, followed by treatment with $25 \mu \mathrm{M}$ chrysin and $5 \mu M$ sorafenib for 2 days. Cell viability was then examined by crystal violet staining (left panel) and statistically quantified, and protein expressions of c-RAF and MEK1 were examined by western blot (right panel). Significantly different at $* p<0.05$, and $* * * p<0.001$.

medium with different serum percentages. As predicted, Hep3B and HepG2 cells became more sensitive to sorafenib treatment when the percentage of serum in cell culture medium was gradually reduced (Figure 3D). We further examined the effect of chrysin on synergy of sorafenib activity in cells maintained with culture medium containing different serum percentages. Since the growth pattern induced by culture medium containing $1 \%$ serum is similar to that 

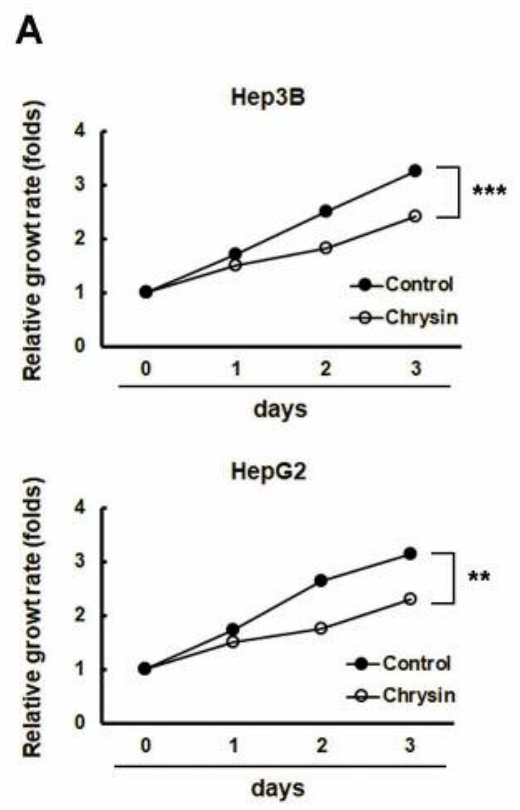

B

\begin{tabular}{cccc}
\multicolumn{2}{c}{ Hep3B } & & \multicolumn{3}{c}{ HepG2 } \\
\hline & FBS $(\%)$ & \\
\hline 10 & 1 & 10 & 1 \\
\hline & & & \\
\hline
\end{tabular}

C

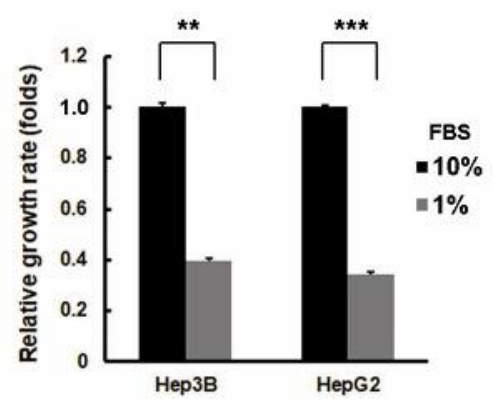

D
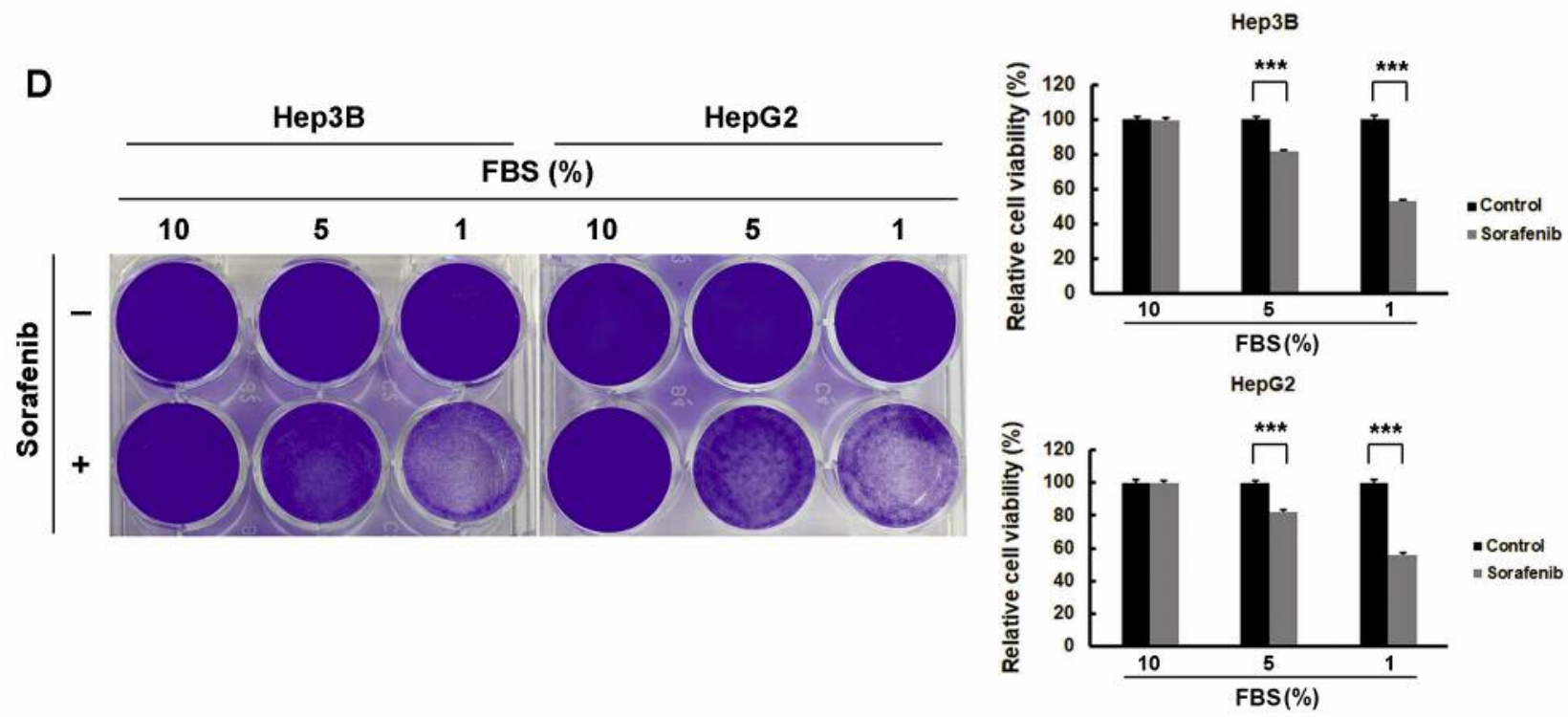

Figure 3. Chrysin-mediated sustained phosphorylation of extracellular signal-regulated protein kinases 1 and 2 (ERK1/2) led to a declining growth pattern conferring sensitivity of hepatocellular carcinoma (HCC) cells to sorafenib treatment. A: Hep3B and HepG2 cells were seeded at a density of $5 \times 10^{3}$ cells/well, followed by treatment with or without $25 \mu \mathrm{M}$ chrysin. Relative cell growth rate was examined at day 1,2, and 3 by MTT assay and statistically quantified. B: Hep $3 B$ and HepG2 cells were seeded at a density of $1 \times 10^{4}$ cells/well and maintained in culture medium with $10 \%$ or $1 \%$ serum. Three days later, cell growth was examined by crystal staining (left panel) and statistically quantified (right panel). (C) Hep3B and HepG2 cells were seeded at a density of $1 \times 10^{5}$ cells/well and maintained in culture medium with $10 \%$ or $1 \%$ serum. Three days later, whole cell lysates were harvested and subjected to western blot for ERK1/2 phosphorylation. D: Hep $3 B$ and HepG2 cells were seeded a density of $1 \times 10^{5}$ cells/well and maintained in culture medium with 10\%, 5\% or 1\% serum. The next day, cells were treated with $5 \mu$ M sorafenib for 2 days. Cell viability was examined by crystal staining (left panel) and statistically quantified (right panel). Significantly different at $* * p<0.01$ and $* * * p<0.001$.

induced by chrysin treatment, sorafenib induced dramatic inhibitory effect on cell viability of Hep3B cells maintained in culture medium with $1 \%$ serum (Figure 4A, upper panel). Co-treatment of chrysin thus hardly enhanced the synergistic effect on sorafenib-mediated viability inhibition (Figure 4A, upper panel). Similar results were obtained in HepG2 cells (Figure 4B). Taken together, our findings show chrysin induced sustained phosphorylation of ERK1/2 and further 
A
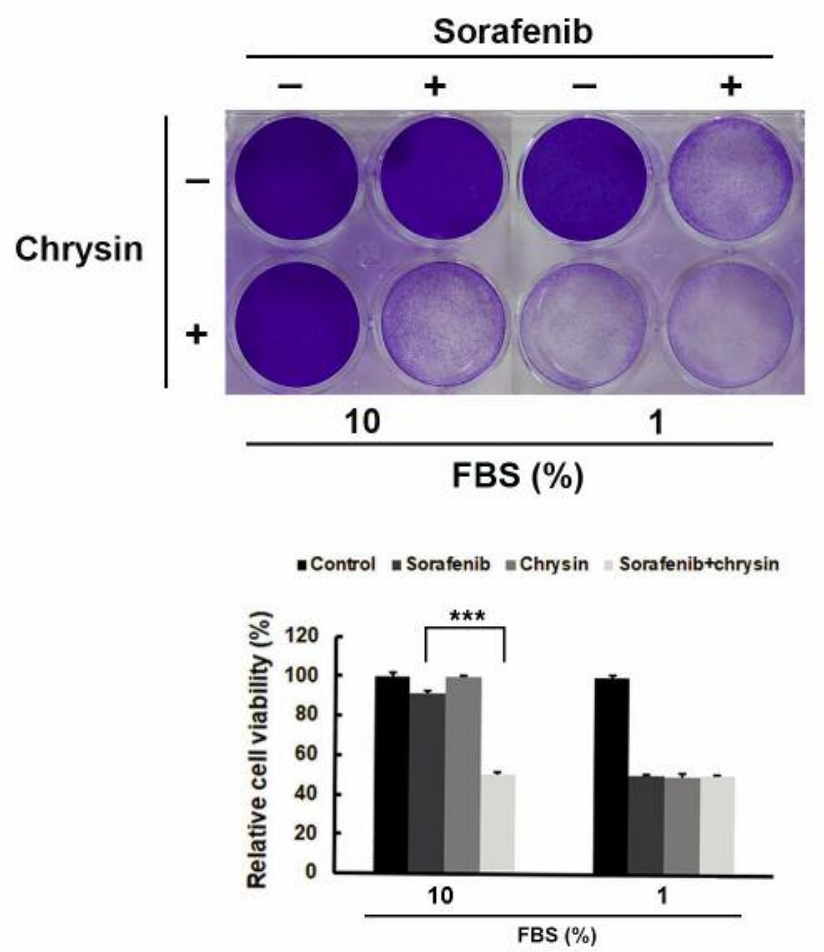

B

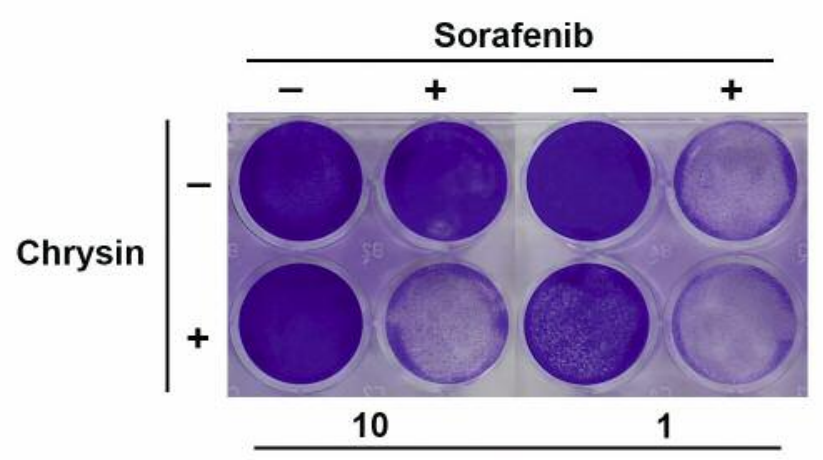

FBS (\%)

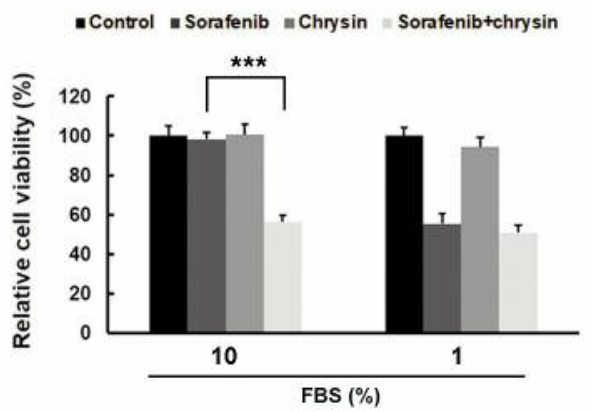

Figure 4. Co-treatment of chrysin did not further enhance the synergistic effect on sorafenib-mediated inhibition of viability in hepatocellular carcinoma (HCC) cells maintained in culture medium with $1 \%$ serum. Hep3B (A) and HepG2 (B) cells were seeded at a density of $1 \times 10^{5}$ cells/well and maintained in culture medium with $10 \%$ or $1 \%$ serum. The next day, cells were treated with $25 \mu M$ chrysin and $5 \mu M$ sorafenib for 2 days. Cell viability was examined by crystal staining (upper panel) and statistically quantified (lower panel). The relative cell viability in the group treated with vehicle alone was set at $100 \%$. *** Significantly different at $p<0.001$.

made HCC cells be in a declining growth state, eventually resulting in a better response to sorafenib treatment.

\section{Discussion}

In this study, we found that chrysin induced sustained phosphorylation of ERK $1 / 2$ and in turn enhanced sorafenibmediated inhibition of cell viability, unraveling another mechanism underlying chrysin-mediated synergy of sorafenib activity. Our study also supports previous findings that a higher basal level of ERK1/2 phosphorylation predicts better response to sorafenib in vitro and in vivo (14-16), implying the importance of ERK1/2 phosphorylation level. A strategy to elevate the level of ERK1/2 phosphorylation may be thus a promising way to enhance sorafenib efficacy.

On the other hand, sorafenib, due to its ability to inhibit tumor cell proliferation and angiogenesis, was approved by the US Food and Drug Administration for the treatment of advanced renal cell carcinoma in 2006, and as the only standard targeted therapy for advanced HCC in 2007. However, the therapeutic results have not been those observed in RCC (3, 4). Many studies explored the mechanisms underlying sorafenib resistance in HCC. For example, activation of epidermal growth factor receptor, signal transducer and activator of transcription 3 as well as ABCG2 identified in our previous study are indicated as determinants of sorafenib sensitivity $(7,21-23)$. Selection of patients with HCC using these predictive determinants for sorafenib treatment or development of combination therapy with sorafenib may be promising strategies for increasing sorafenib efficacy. Since the mechanisms underlying sorafenib resistance are diverse, development of a drug overcoming multiple resistance mechanisms will be the best way to effectively enhance sorafenib efficacy. Chrysin is one such candidate. In our previous study, co-treatment of HCC cells with chrysin greatly augmented the anti-tumor activity of sorafenib through inhibiting transporter activity of ABCG2 (7). In this study, we demonstrate that chrysin induced sustained phosphorylation of ERK1/2 and rendered HCC cells to exhibit a declining growth pattern, which in turn made HCC cells more sensitive to sorafenib treatment (Figures 1-3). Therefore, chrysin may be applied as an 
effective sensitizer to increase sorafenib efficacy through multiple mechanisms at the same time, which awaits further validation in the clinical scenario.

\section{Conflicts of Interest}

All the Authors declare that they have no conflicts of interest in regard to this study.

\section{Acknowledgements}

This work was supported by grants from E-Da Hospital (EDPJ105083 and EDAHP107053).

\section{References}

1 Wilhelm SM, Carter C, Tang L, Wilkie D, McNabola A, Rong H, Chen C, Zhang X, Vincent P, McHugh M, Cao Y, Shujath J, Gawlak S, Eveleigh D, Rowley B, Liu L, Adnane L, Lynch M, Auclair D, Taylor I, Gedrich R, Voznesensky A, Riedl B, Post LE, Bollag G and Trail PA: Bay 43-9006 exhibits broad spectrum oral antitumor activity and targets the RAF/MEK/ERK pathway and receptor tyrosine kinases involved in tumor progression and angiogenesis. Cancer Res 64(19): 7099-7109, 2004.

2 Carlomagno F, Anaganti S, Guida T, Salvatore G, Troncone G, Wilhelm SM and Santoro M: Bay 43-9006 inhibition of oncogenic RET mutants. J Natl Cancer Inst 98(5): 326-334, 2006.

3 Llovet JM, Ricci S, Mazzaferro V, Hilgard P, Gane E, Blanc JF, de Oliveira AC, Santoro A, Raoul JL, Forner A, Schwartz M, Porta C, Zeuzem S, Bolondi L, Greten TF, Galle PR, Seitz JF, Borbath I, Haussinger D, Giannaris T, Shan M, Moscovici M, Voliotis D, Bruix J and SHARP Investigators Study Group: Sorafenib in advanced hepatocellular carcinoma. N Engl J Med 359(4): 378-390, 2008.

4 Cheng AL, Kang YK, Chen Z, Tsao CJ, Qin S, Kim JS, Luo R, Feng J, Ye S, Yang TS, Xu J, Sun Y, Liang H, Liu J, Wang J, Tak WY, Pan H, Burock K, Zou J, Voliotis D and Guan Z: Efficacy and safety of sorafenib in patients in the Asia-Pacific region with advanced hepatocellular carcinoma: A phase III randomised, double-blind, placebo-controlled trial. Lancet Oncol 10(1): 25-34, 2009.

5 Perez-Tomas R: Multidrug resistance: Retrospect and prospects in anticancer drug treatment. Curr Med Chem 13(16): 1859$1876,2006$.

6 Glavinas H, Krajcsi P, Cserepes J and Sarkadi B: The role of $\mathrm{ABC}$ transporters in drug resistance, metabolism and toxicity. Curr Drug Deliv 1(1): 27-42, 2004.

7 Huang WC, Hsieh YL, Hung CM, Chien PH, Chien YF, Chen LC, Tu CY, Chen $\mathrm{CH}$, Hsu SC, Lin YM and Chen YJ: BCRP/ABCG2 inhibition sensitizes hepatocellular carcinoma cells to sorafenib. PLoS One 8(12): e83627, 2013.

8 Garcia-Salas P, Morales-Soto A, Segura-Carretero A and Fernandez-Gutierrez A: Phenolic-compound-extraction systems for fruit and vegetable samples. Molecules 15(12): 8813-8826, 2010.

9 Martos I, Ferreres F and Tomas-Barberan FA: Identification of flavonoid markers for the botanical origin of eucalyptus honey. J Agric Food Chem 48(5): 1498-1502, 2000.
10 Li-Weber M: Targeting apoptosis pathways in cancer by Chinese medicine. Cancer Lett 332(2): 304-312, 2013.

11 Kasala ER, Bodduluru LN, Madana RM, V AK, Gogoi R and Barua CC: Chemopreventive and therapeutic potential of chrysin in cancer: Mechanistic perspectives. Toxicol Lett 233(2): 214225, 2015.

12 Khoo BY, Chua SL and Balaram P: Apoptotic effects of chrysin in human cancer cell lines. Int J Mol Sci 11(5): 2188-2199, 2010.

13 Lin YM, Chen CI, Hsiang YP, Hsu YC, Cheng KC, Chien PH, Pan HL, Lu CC and Chen YJ: Chrysin attenuates cell viability of human colorectal cancer cells through autophagy induction unlike 5-fluorouracil/oxaliplatin. Int J Mol Sci 19(6), 2018. doi: 10.3390/ijms 19061763.

14 Abou-Alfa GK, Schwartz L, Ricci S, Amadori D, Santoro A, Figer A, De Greve J, Douillard JY, Lathia C, Schwartz B, Taylor I, Moscovici M and Saltz LB: Phase II study of sorafenib in patients with advanced hepatocellular carcinoma. J Clin Oncol 24(26): 4293-4300, 2006.

15 Zhang Z, Zhou X, Shen $H$, Wang $D$ and Wang $Y$ : Phosphorylated ERK is a potential predictor of sensitivity to sorafenib when treating hepatocellular carcinoma: Evidence from an in vitro study. BMC Med 7: 41, 2009.

16 Liang Y, Chen J, Yu Q, Ji T, Zhang B, Xu J, Dai Y, Xie Y, Lin $\mathrm{H}$, Liang $\mathrm{X}$ and Cai $\mathrm{X}$ : Phosphorylated ERK is a potential prognostic biomarker for sorafenib response in hepatocellular carcinoma. Cancer Med 6(12): 2787-2795, 2017.

17 Sewing A, Wiseman B, Lloyd AC and Land H: High-intensity RAF signal causes cell cycle arrest mediated by p21CIP1. Mol Cell Biol 17(9): 5588-5597, 1997.

18 Woods D, Parry D, Cherwinski H, Bosch E, Lees E and McMahon M: RAF-induced proliferation or cell cycle arrest is determined by the level of RAF activity with arrest mediated by p21CIP1. Mol Cell Biol 17(9): 5598-5611, 1997.

19 Marshall CJ: Specificity of receptor tyrosine kinase signaling: Transient versus sustained extracellular signal-regulated kinase activation. Cell 80(2): 179-185, 1995.

20 Stork PJ: ERK signaling: Duration, duration, duration. Cell Cycle 1(5): 315-317, 2002.

21 Sun T, Liu H and Ming L: Multiple roles of autophagy in the sorafenib resistance of hepatocellular carcinoma. Cell Physiol Biochem 44(2): 716-727, 2017.

22 Zhu YJ, Zheng B, Wang HY and Chen L: New knowledge of the mechanisms of sorafenib resistance in liver cancer. Acta Pharmacol Sin 38(5): 614-622, 2017.

23 Chen J, Jin R, Zhao J, Liu J, Ying H, Yan H, Zhou S, Liang Y, Huang D, Liang X, Yu H, Lin H and Cai X: Potential molecular, cellular and microenvironmental mechanism of sorafenib resistance in hepatocellular carcinoma. Cancer Lett 367(1): 1-11, 2015.
Received December 3, 2018

Revised December 13, 2018

Accepted December 14, 2018 